\title{
IL-36r in Enthesitis related Juvenile Idiopathic Arthritis and its association with disease activity.
}

\author{
Sanjukta Majumder et al. ${ }^{1}$ \\ ${ }^{1}$ Affiliation not available
}

September 13, 2021

\begin{abstract}
IL-36 has been implicated in the pathogenesis of spondyloarthropathies (SpA) like psoriasis and inflammatory bowel disease. Enthesitis related arthritis (ERA) category of juvenile idiopathic arthritis is a form of juvenile SpA, however no data is available on the role of IL-36 in this disease. IL-36 $\alpha, \beta, \gamma$ and IL-36R mRNA expression in blood and synovial fluid mononuclear cells and IL-36 $\alpha, \gamma$, IL-36Ra, IL-6 and IL-17 levels were measured in serum and synovial fluid (SF). IL-36 $\gamma$ production by fibroblast like synoviocytes (FLS) by pro-inflammatory cytokines and its effect on FLS was also studied.mRNA levels of IL-36 $\alpha$, IL-36r and IL-36R were increased in PBMCs of ERA patients as compared to healthy controls however only IL-36r was measurable in serum of one third of patients. In SFMCs, all 4 mRNA were detectable but were lower than RA patients. SF IL-36r levels correlated with disease activity score $(\mathrm{r}=0.51, \mathrm{p}<0.0001)$, SF IL-6 $(\mathrm{r}=0.4, \mathrm{p}=0.0063)$ and IL-17 levels $(\mathrm{r}=0.57, \mathrm{p}=0.0018)$. Pro-inflammatory cytokines increased expression of IL-36 $\gamma$ and IL-6 in FLS cultures. SFs from 5 ERA patients also increased expressions of IL-36r and IL-6 in FLS which could be blocked by using IL-36Ra.This suggests that pro-inflammatory cytokines aid in upregulation of IL-36 $\gamma$ which in turn upregulates expression of IL-6. This might lead to a positive feedback loop of inflammation in ERA. Association of SF levels of IL-36r with disease activity further supports this possibility. IL-36Ra based therapy may have a role in ERA.
\end{abstract}

Funding support: The work was supported by the National postdoctoral fellowship to Sanjukta Majumder from Department of Science and Technology- Science and Engineering Research Board, Govt. of India.

\section{Introduction}

Juvenile Idiopathic Arthritis (JIA) is the most common form of childhood rheumatic diseases and encompasses a heterogeneous group of arthritic diseases. Among its different subtypes, Enthesitis Related Arthritis (JIA-ERA) is the most common form of JIA in south-east Asia including India and constitutes 30-35\% of patients, in contrast to only 5-8\% in Western countries. It resembles adult spondyloarthropathies (SpA) with which it shares male preponderance, presence of enthesitis, lower limb arthritis, sacroiliitis and HLA B27 association where HLA B27 prevalence varies from $60-90 \%$ in different forms of SpA [1, 2].

Although pathogenesis of JIA-ERA is unclear, recent data shows evidence of gut dysbiosis suggesting a role for interaction between HLA B27 and gut microbiome. Gut microbes, by activating TLR mediated pathway and HLA B27 induce an unfolded protein response (UPR) that results in activation of monocyte -macrophage lineage cells. This leads to production of pro-inflammatory cytokines including IL-23 by these cells. IL-23 in turn acts on IL-23R bearing T cells, innate lymphoid cells and NK cells to cause production of IL-17 and IL-22, the two key cytokines involved in inflammation in joints and enthesitis. In addition, other pro-inflammatory cytokines like IL-6, TNF-alpha, IL-8 and IL-15 are also produced which enhance the inflammation [3-10]. 
Recently, the IL-1 family member IL-36 (having isoforms $\alpha, \beta$ and $\gamma$ ) and its receptor antagonist IL-36Ra have also been implicated in the pathogenesis of psoriasis and inflammatory bowel disease (IBD) both of which can precipitate SpA. Psoriatic skin lesions show upregulation of all 3 isoforms of IL-36, and keratinocytes derived from psoriatic lesion produce IL-36 in response to TNF- $\alpha$, IL-17 and IL-22. Mice over-expressing IL-36 $\alpha$ develop psoriasis like skin lesions and deletion of IL-36Ra gene in them increases disease severity $[11,12]$. Synovial lining in patients with psoriatic arthritis (PsA) as well as rheumatoid arthritis (RA) also show higher expression of IL-36 compared to osteoarthritis [13-17]. The synovial plasma cells were identified as the primary source of IL-36 expression, which in turn induced production of inflammatory cytokines by fibroblast like synoviocytes (FLS) [11]. In animal models of psoriasis, blockage of IL-36R results in disease amelioration accompanied by reduction in IL-17, leukocyte infiltration and keratinocyte activation [18-20]. Most importantly, IL-17 stimulates keratinocytes to produce IL-36 which in turn induce IL-17 production leading to a positive feed-back loop in psoriasis [21], possibly resulting in the dysregulated state of immune response. However, in RA, IL-36 expression does not seem to correlate with IL-17 signal [11, 17].

As mentioned above, JIA-ERA, IBD and psoriasis belong to the broad group of SpA, but not RA since the synovitis in RA is different from that in SpA. We therefore hypothesized that, like PsA and IBD, IL-36 may also be associated with synovitis in JIA-ERA and contribute to the dysregulated inflammatory response of the synovium, accompanied by production of other pro-inflammatory cytokines.

\section{Materials and Methods}

\section{Patients}

Fifteen ERA patients fulfilling the JIA-International League of Associations for Rheumatology (ILAR) classification criteria [22] were included in the study. None of the patients had psoriasis or IBD. Clinical details were collected, and disease activity was assessed using Juvenile Spondyloarthropathy Disease Activity Score (JSpaDA) by the treating rheumatologist at the time of sample collection. As controls, blood from 10 young adult healthy males and blood and synovial fluid (SF) from 5 patients with RA undergoing joint aspiration were also obtained. Approval from the Institutional Ethics Committee was obtained prior to commencement of the study (IEC approval code: 2018-10-EMP-102) and all patients/guardians provided written informed consent.

\section{Isolation of PBMCs and SFMCs}

Peripheral blood mononuclear cells (PBMC) and synovial fluid mononuclear cells (SFMC) were isolated by density gradient centrifugation. Briefly, diluted heparinized blood or diluted pelleted synovial fluid cells were layered on Histopaque 1077 (Sigma-Aldrich, St Louis, MO, USA) and centrifuged (1800 rpm, $30 \mathrm{~min}$ ). The interface containing mononuclear cells was collected and washed (x3) with PBS.

\section{Fibroblast like synoviocyte (FLS) culture}

SF was collected from JIA-ERA patient in a heparinized tube and centrifuged (1800 rpm, $10 \mathrm{~min}$ ). Cell pellet was re-suspended in Dulbecco's modified Eagle's medium (DMEM) containing $10 \%$ fetal bovine serum (FBS) and plated in a tissue culture flask. Cells were incubated at $37{ }^{\circ} \mathrm{C}$ in $5 \% \mathrm{CO}_{2}$ for $48 \mathrm{~h}$. Medium was aspirated and the cell monolayer was washed with PBS to remove any non-adherent cell prior to addition of fresh medium.

FLS cultures from synovial biopsies were done as previously described [23]. Briefly, perioperatively obtained synovial tissues from patients undergoing joint replacement $(n=4)$ were finely minced and treated with collagenase A $\left(37{ }^{0} \mathrm{C}, 90 \mathrm{~min}\right)$. After removing collagenase, cells were washed (x3) with PBS and allowed to adhere $(48 \mathrm{~h})$ in cell culture flasks. Non-adherent cells were removed with PBS washes and fresh complete medium (DMEM+5\% FBS) was added. Medium was replaced every 3-4 days and routinely split after reaching confluency. All experiments were performed when FLS were between $4^{\text {th }}-6^{\text {th }}$ passages.

For Gene expression studies, FLS were seeded in 6 -well culture plates $\left(2 \times 10^{5}\right.$ cells/well $)$ and allowed to adhere for $24 \mathrm{~h}$. Cells were initially starved for serum $(2 \mathrm{~h})$ and then stimulated for 3,6 or $24 \mathrm{~h}$ with 


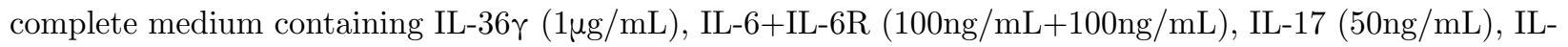
$6+\mathrm{IL}-6 \mathrm{R}+\mathrm{IL}-17$, TNF- $\alpha(50 \mathrm{ng} / \mathrm{mL})$ or medium alone. All recombinant protein concentrations were used as previously reported in various studies.

To study the effect of SF from JIA-ERA patients on FLS, cells were stimulated with SF from 5 JIA-ERA patients having detectable levels of IL-36r (10\% SF in complete medium), in the presence or absence of IL36Ra $(1 \mu \mathrm{g} / \mathrm{mL})$. All stimulations were done in duplicates. Post-stimulation, cells were harvested and stored in Trizol for RNA extraction. RT-qPCR was performed with the cDNA reverse transcribed from isolated RNA samples.

For ELISA, FLS were seeded in 96 -well plates $\left(10^{4}\right.$ cells/well $)$ and stimulated $(8 \mathrm{~h}$, in triplicate) with the stimulants as previously described. Later, stimulants were removed, and cells were washed (x3) with PBS. Cells were replenished with fresh complete medium and allowed to rest overnight. Supernatants were stored and cell viability was assessed using MTT assay.

\section{ELISA}

Clotted blood and SF were centrifuged (1800 rpm, $10 \mathrm{~min}$ ) and serum and cell-free SF was stored at -80 ${ }^{0} \mathrm{C}$ in aliquots. ELISAs for IL-36 $\alpha$, IL-36 $\gamma$, IL-36 Ra, IL-6 and IL-17A were performed using ELISA kits (R\&D Systems, USA or BD Biosciences, USA). All ELISAs were performed by following the manufacturers' protocols.

\section{RNA isolation, cDNA synthesis and gene expression studies using RT-qPCR}

RNA was isolated from PBMCs and SFMCs by Trizol-Chloroform extraction method and cDNA was synthesized utilizing the High-Capacity cDNA synthesis kit (Invitrogen, USA). Real Time PCR was performed using SYBR Green Master mix (Invitrogen, USA). Primer sets used for RT-qPCR are listed in Table 1. All RT-qPCR analyses were performed in the Roche Light Cycler 480 instrument (Roche, USA). Melting curve analyses were performed for every experiment to rule out non-specific mRNA amplification. Fold change calculation was done using the $\Delta \Delta \mathrm{Ct}$ method. Results were denoted either by fold difference (compared to healthy control) or difference in $\mathrm{Ct}$ values $(\Delta \mathrm{Ct})$ of gene of interest and housekeeping genes.

\section{Statistical analyses}

The data was analyzed using Graph Pad Prism 8.0 Software (GraphPad Software Inc. San Diego, CA, USA). All cytokine levels (protein and mRNA) are expressed as median and interquartile range (IQR). Nonparametric Mann-Whitney U test and Wilcoxon signed-rank test were used for intergroup comparisons. Correlation analysis was performed using Spearman's rank correlation test. A P value of $<0.05$ was considered statistically significant.

\section{Results}

\section{Clinical characteristics of JIA-ERA patients}

For mRNA expression studies, cells from the enrolled JIA-ERA patients and controls (RA patients and healthy subjects) were used. In addition, for SF and serum studies, previously stored samples were also included. A total of 84 JIA-ERA, 20 RA and 24 healthy subject samples were used for ELISA, out of which 48 were paired samples (both serum and SF). Among the 84 JIA-ERA patients, 77 were boys. Their age was $17 \pm 5$ years and disease duration were $60 \pm 72$ months. All had active disease with ESR of $57.5 \pm 55.5 \mathrm{~mm}$ and JSpaDA of $3.5 \pm 2$ (Table 2). Age of healthy subjects was $19 \pm 1$ years. Out of 84 JIA-ERA patients, 21 were on disease modifying anti rheumatic drugs (DMARDs) and the rest were treated with non-steroidal anti-inflammatory drugs (NSAIDs) or intra-articular corticosteroids.

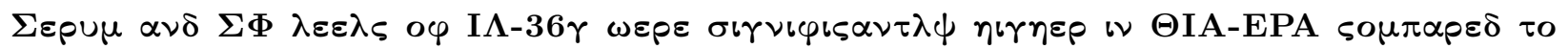
$\eta \varepsilon \alpha \lambda \tau \eta \psi \sigma \cup \beta \vartheta \varepsilon \varsigma \tau \varsigma$

Serum IL-36r was detected in 21 of 61 JIA-ERA patients (median \pm IQR, $0 \pm 42.1 \mathrm{pg} / \mathrm{mL}$ ) and only 1 of 24 healthy subjects $(0 \pm 0)(\mathrm{p}<0.01$, Figure $1 \mathrm{~A})$. In $\mathrm{SF}$, it was present in 24 of 75 JIA-ERA samples $(0 \pm$ 
$26.78 \mathrm{pg} / \mathrm{mL}$, Figure 1B). However, IL-36 $\gamma$ levels in the serum $(198.2 \pm 672.9 \mathrm{pg} / \mathrm{mL})$ as well as SF (460 $\pm 1345 \mathrm{pg} / \mathrm{mL}$ ) of RA patients were significantly higher than JIA-ERA and healthy subjects, possibly as patients with RA have higher degree of synovial inflammation. IL-36 $\alpha$ was detected in the serum and SF of only one JIA-ERA patient and none of the healthy sera. IL-36Ra was also not found in any sample (data not shown). In paired samples, serum and SF levels of IL-36 $\gamma$ showed a positive correlation $(\mathrm{r}=0.32, \mathrm{p}<$ 0.0001, Figure 1C, D), but were not significantly different from each other.

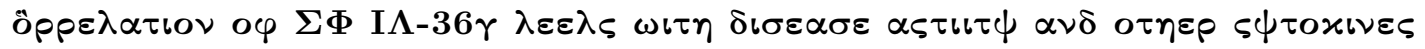

Patients with JIA-ERA had significantly higher serum and SF levels of IL-6 and IL-17 than the healthy subjects (Figure 2A, B). In patients, SF levels of both cytokines were significantly higher than the serum level (Figure 2C). Serum IL-36 $\gamma$ levels did not show any correlation with any clinical parameter. However, SF IL-36 $\gamma$ levels correlated positively with IL-6 and IL-17 levels $(r=0.40, p=0.0063$ and $r=0.57, p=0.0018$, respectively; Figure 2D, E). IL-36r levels in SF also showed positive correlation with disease activity score JSpaDA $(r=0.51, \mathrm{p}<0.0001$; Figure $2 \mathrm{~F})$. Patients who were undergoing treatment with either methotrexate or sulfasalazine had significantly lower SF levels of IL-36 $\gamma$ than the patients who were not under treatment with any DMARD $(\mathrm{p}<0.05)$. However, serum level of IL-36r did not vary between the two groups (Figure $3 \mathrm{~A}, \mathrm{~B})$.

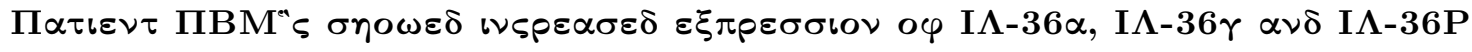

PBMCs of JIA-ERA patients $(\mathrm{n}=15)$ had higher levels of IL-36 $\alpha$, IL-36 $\gamma$ and IL-36R mRNA as compared to healthy controls $(n=10)$ and no difference was observed in the expression levels of IL-36 $\beta$ (Figure 4A). However, mRNA expression of all 4 genes were observed in in SFMCs of JIA-ERA patients, but was lower than that seen in RA (Figure 4B).

\section{Effect of pro-inflammatory cytokines on FLS}

Stimulation of FLS with pro-inflammatory cytokines increased the mRNA expression of both IL-6 and IL-36 $\gamma$ (Figure 5A, B). Stimulation with IL-36ץ induced IL-6 mRNA as well as protein production (Figure 5C). No expression of IL-17 at RNA or protein level was observed during any treatments (data not shown).

\section{Effect of SF from patients with JIA-ERA on FLS and impact of IL-36Ra treatment}

Stimulation of FLS with SF from 5 JIA-ERA patients (who had detectable levels of IL-36r) enhanced the mRNA expression of both IL-6 and IL-36 $\gamma$ as compared to controls. However, in presence of IL-36Ra, the SF induced expression of both genes was significantly reduced ( $<<0.0001$; Figure 6A, B). IL-6 protein expression was also induced in presence of SF and reduced significantly when stimulated in presence of IL$36 \mathrm{Ra}(\mathrm{p}<0.0001$; Figure 6C). This further strengthened the notion that IL-36 $\gamma$ can induce the expression of pro-inflammatory cytokines like IL-6 as well as of IL-36 $\gamma$ itself.

\section{Discussion}

JIA-ERA patients had higher expression of IL-36 $\gamma$ as well as higher serum levels compared to healthy controls. Further SF IL-36 $\gamma$ correlated with SF IL-17 and IL-6 levels as well as with disease activity. Our finding of enhanced IL-36r expression in PBMC and its higher serum levels in JIA-ERA is similar to that reported for other rheumatic diseases including PsA [24]. IL-36 $\alpha$ and IL-36 $\gamma$ were found significantly increased in SLE patients with arthritis compared to those without arthritis [25]. Increased expression of IL-36 was also seen in patients with RA as well as various mouse models of RA [11, 15, 17, 24, 26]. Patients treated with DMARDs had lower SF IL-36r levels than those who were not on DMARDs. In psoriasis, arthritis and SLE also, the expression of IL-36 cytokines has correlated significantly with disease activity $[11,15,25,27]$. In psoriatic arthritis, patients who did not respond to DMARD treatment had increased expression of IL-36 $\alpha$ in their synovium [24].

Higher levels of IL-36r in SF and its correlation with disease activity and synovial IL-6 and IL-17 levels may suggests it's importance in local milieu. Skin biopsies of psoriasis patients, intestinal tissue of IBD patients and urine of lupus nephritis patients have also shown high localized expression of IL-36. In addition, studies 
have shown that IL-36 is upregulated in the synovium of PsA and RA patients, and synovial CD138+ plasma cells express IL-36 [13, 14, 17, 24, 27, 28].

Positive correlation of SF IL-36 $\gamma$ with IL-6 and IL-17 levels could also suggest that all 3 proinflammatory cytokines were produced in response to the same stimuli. Alternatively, IL-36 may enhance the production of IL-17 and IL-6. In this context, we did observe increased expression of mRNA as well as IL-6 production by FLS upon exposure to IL-36 $\gamma$, but not IL-17. In a previous study, IL36 $\alpha$ was shown to induce IL-6 and IL-8 production in synovial fibroblasts [24]. IL-36 cytokines were also found to induce production of IL-6 in human colonic subepithelial myofibroblasts [17, 29, 30] further strengthens our finding.

The in vitro effects of SF from JIA-ERA on FLS suggested that IL-36 $\gamma$ could induce the production of IL-6 and IL-36 $\gamma$ itself, but not IL-17. SF-induced production of IL-6 and IL-36 $\gamma$ could be inhibited by IL-36Ra (IL-36R antagonist) suggesting that IL-36 could be associated with synovial inflammation in JIA-ERA. Besides IL-17, IL-6 is also an important cytokine associated with synovial inflammation of JIA-ERA [5]. In human blood monocyte-derived dendritic cells (BMDCs) IL-36Ra increased LPS-induced IL-6 production however reduced IL-8, IL-17 and IL-22 production in PBMCs induced by IL-36r [31]. Boutet et al have shown that IL-36 $\alpha$-induced production of IL- 8 from FLS is significantly reduced in presence of IL-36Ra [24] and IL-36 $\gamma$-induced production of IL- 8 by human PBMCs was also reduced by IL-36Ra. However, to the best of our knowledge, no previous studies have reported reduced IL-6 or IL-36 production in presence of IL-36Ra.

To conclude, our results suggest that, among the IL-36 family of pro-inflammatory cytokines, IL-36r may have a role in the synovial inflammation seen in JIA-ERA patients. And as Spesolimab and Imsidolimab, the IL-36 receptor antibodies are currently undergoing clinical trials and showing promise in treatment of general pustular psoriasis and palmoplantar pustulosis [16, 18, 32, 33], targeting IL-36 with antibodies to IL-36 receptor may also prove beneficial to the patients with JIA-ERA pending further investigation.

\section{References}

1. Ravelli, A. and A. Martini, Juvenile idiopathic arthritis.Lancet, 2007. 369 (9563): p. 767-778.

2. Aggarwal, A. and D.P. Misra, Enthesitis-related arthritis.Clin Rheumatol, 2015. 34 (11): p. 1839-46.

3. Bridgewood, C., et al., Interleukin-23 pathway at the enthesis: The emerging story of enthesitis in spondyloarthropathy. Immunol Rev, 2020. 294 (1): p. 27-47.

4. Agarwal, S., R. Misra, and A. Aggarwal, Interleukin 17 levels are increased in juvenile idiopathic arthritis synovial fluid and induce synovial fibroblasts to produce proinflammatory cytokines and matrix metalloproteinases. J Rheumatol, 2008. 35 (3): p. 515-9.

5. Mahendra, A., R. Misra, and A. Aggarwal, Th1 and Th17 Predominance in the Enthesitis-related Arthritis Form of Juvenile Idiopathic Arthritis. J Rheumatol, 2009. 36 (8): p. 1730-6.

6. Majumder, S. and A. Aggarwal, Juvenile idiopathic arthritis and the gut microbiome: Where are we now? Best Pract Res Clin Rheumatol, 2019. 33 (6): p. 101496.

7. DeLay, M.L., et al., HLA-B27 misfolding and the unfolded protein response augment interleukin-23 production and are associated with Th17 activation in transgenic rats. Arthritis Rheum, 2009.60 (9): p. 2633-43.

8. Colbert, R.A., et al., HLA-B27 misfolding and spondyloarthropathies. Prion, 2009. 3 (1): p. 15-26.

9. Smith, J.A. and R.A. Colbert, Review: The interleukin-23/interleukin-17 axis in spondyloarthritis pathogenesis: Th17 and beyond. Arthritis Rheumatol, 2014. 66 (2): p. 231-41.

10. Saxena, N., A. Aggarwal, and R. Misra, Elevated concentrations of monocyte derived cytokines in synovial fluid of children with enthesitis related arthritis and polyarticular types of juvenile idiopathic arthritis. J Rheumatol, 2005. 32 (7): p. 1349-53. 
11. Boutet, M.A., et al., Distinct expression of interleukin (IL)-36alpha, beta and gamma, their antagonist $I L-36 R a$ and IL-38 in psoriasis, rheumatoid arthritis and Crohn's disease. Clin Exp Immunol, 2016. 184 (2): p. 159-73.

12. Towne, J.E. and J.E. Sims, IL-36 in psoriasis. Curr Opin Pharmacol, 2012. 12 (4): p. 486-90.

13. Leon, G., S. Hussey, and P.T. Walsh, The Diverse Roles of the IL-36 Family in Gastrointestinal Inflammation and Resolution. Inflamm Bowel Dis, 2021. 27 (3): p. 440-450.

14. Fonseca-Camarillo, G., et al., Differential Expression of IL-36 Family Members and IL-38 by Immune and Nonimmune Cells in Patients with Active Inflammatory Bowel Disease. Biomed Res Int, 2018.2018 : p. 5140691.

15. Boutet, M.A., A. Nerviani, and C. Pitzalis, IL-36, IL-37, and IL-38 Cytokines in Skin and Joint Inflammation: A Comprehensive Review of Their Therapeutic Potential. Int J Mol Sci, 2019. 20 (6).

16. Buhl, A.L. and J. Wenzel, Interleukin-36 in Infectious and Inflammatory Skin Diseases. Front Immunol, 2019. 10 : p. 1162.

17. Frey, S., et al., The novel cytokine interleukin-36alpha is expressed in psoriatic and rheumatoid arthritis synovium. Ann Rheum Dis, 2013. 72 (9): p. 1569-74.

18. Iznardo, H. and L. Puig, Exploring the Role of IL-36 Cytokines as a New Target in Psoriatic Disease. Int J Mol Sci, 2021.22 (9).

19. Hovhannisyan, Z., et al., Enhanced IL-36R signaling promotes barrier impairment and inflammation in skin and intestine. Sci Immunol, 2020. 5 (54).

20. Mahil, S.K., et al., An analysis of IL-36 signature genes and individuals with IL1RL2 knockout mutations validates $I L-36$ as a psoriasis therapeutic target. Sci Transl Med, 2017. 9 (411).

21. Pfaff, C.M., et al., The psoriasis-associated IL-17A induces and cooperates with IL-36 cytokines to control keratinocyte differentiation and function. Sci Rep, 2017. 7 (1): p. 15631.

22. Petty, R.E., et al., International League of Associations for Rheumatology classification of juvenile idiopathic arthritis: second revision, Edmonton, 2001. J Rheumatol, 2004. 31 (2): p. 390-2.

23. Rosengren, S., D.L. Boyle, and G.S. Firestein, Acquisition, culture, and phenotyping of synovial fibroblasts. Methods Mol Med, 2007. 135 : p. 365-75.

24. Boutet, M.A., et al., Interleukin-36 family dysregulation drives joint inflammation and therapy response in psoriatic arthritis.Rheumatology (Oxford), 2020. 59 (4): p. 828-838.

25. Mai, S.Z., et al., Increased serum IL-36alpha and IL-36gamma levels in patients with systemic lupus erythematosus: Association with disease activity and arthritis. Int Immunopharmacol, 2018. 58 : p. 103108.

26. Hahn, M., S. Frey, and A.J. Hueber, The novel interleukin-1 cytokine family members in inflammatory diseases. Curr Opin Rheumatol, 2017. 29 (2): p. 208-213.

27. Majumder, S., et al., Elevated urinary IL-36gamma in patients with active lupus nephritis and response to treatment. Lupus, 2021.30 (6): p. 921-925.

28. Ngo, V.L., et al., IL-36 cytokines and gut immunity.Immunology, 2021. 163 (2): p. 145-154.

29. Kanda, T., et al., Interleukin(IL)-36alpha and IL-36gamma Induce Proinflammatory Mediators from Human Colonic Subepithelial Myofibroblasts. Front Med (Lausanne), 2015. 2 : p. 69.

30. Chu, M., et al., Elevated Expression and Pro-Inflammatory Activity of IL-36 in Patients with Systemic Lupus Erythematosus.Molecules, 2015. 20 (10): p. 19588-604. 
31. van de Veerdonk, F.L., et al., IL-38 binds to the IL-36 receptor and has biological effects on immune cells similar to IL-36 receptor antagonist. Proc Natl Acad Sci U S A, 2012. 109 (8): p. 3001-5.

32. Mrowietz, U., et al., Spesolimab, an Anti-Interleukin-36 Receptor Antibody, in Patients with Palmoplantar Pustulosis: Results of a Phase IIa, Multicenter, Double-Blind, Randomized, Placebo-Controlled Pilot Study. Dermatol Ther (Heidelb), 2021. 11 (2): p. 571-585.

33. Ratnarajah, K., et al., Spesolimab: A Novel Treatment for Pustular Psoriasis. J Cutan Med Surg, 2020. 24 (2): p. 199-200.

Figures and Tables

Table 1 : List of primers used for the study.

\begin{tabular}{|c|c|}
\hline Gene Name & Primer Sequence 5'-3' \\
\hline IL-36 $\alpha$ FP & TAGTGGGTGTAGTTCTGTAGTGTGC \\
\hline IL-36 $\alpha$ RP & GTTCGTCTCAAGAGTGTCCAGATAT \\
\hline IL-36 $\beta$ FP & TCTTAGCCGCAGCATTAAGC \\
\hline IL-36 $\beta$ RP & TGCCCTGAATTTCTGCACAG \\
\hline IL-36r FP & AGAGTAACCCCAGTCAGCGTG \\
\hline IL-36r RP & AGGGTGGTGGTACAAATCCAA \\
\hline IL-36R FP & AAACACCTAGCAAAAGCCCAG \\
\hline IL-36R RP & AGACTGCCCGATTTTCCTATG \\
\hline GAPDH FP & GGAGCGAGATCCCTCCAAAAT \\
\hline GAPDH RP & GGCTGTTGTCATACTTCTCATGG \\
\hline IL-6 FP & ACTCACCTCTTCAGAACGAATTG \\
\hline IL-6 RP & CCATCTTTGGAAGGTTCAGGTTG \\
\hline IL-17 FP & CAT CCA TAA CCG GAA TAC CAA TA \\
\hline IL-17 RP & TAG TCC ACG TTC CCA TCA GC \\
\hline$\beta$ - Actin FP & AGAAAATCTGGCACCACACC \\
\hline$\beta$ - Actin FP & AGAGGCGTACAGGGATAGCA \\
\hline
\end{tabular}

Table 2: Clinical characteristics of patients with JIA-ERA. Results denoted as Median \pm IQR where necessary.

\begin{tabular}{ll}
\hline Variables & Values \\
\hline Patients & $84(77 \mathrm{M}+7 \mathrm{~F})$ \\
Age (Years) & $17 \pm 5$ \\
Duration of Disease (Months) & $60 \pm 72$ \\
ESR (mm) & $57.5 \pm 55.5$ \\
Disease activity as measured by JSpaDA & $3.5 \pm 2$ \\
Patients on DMARDs & 21 \\
\hline
\end{tabular}



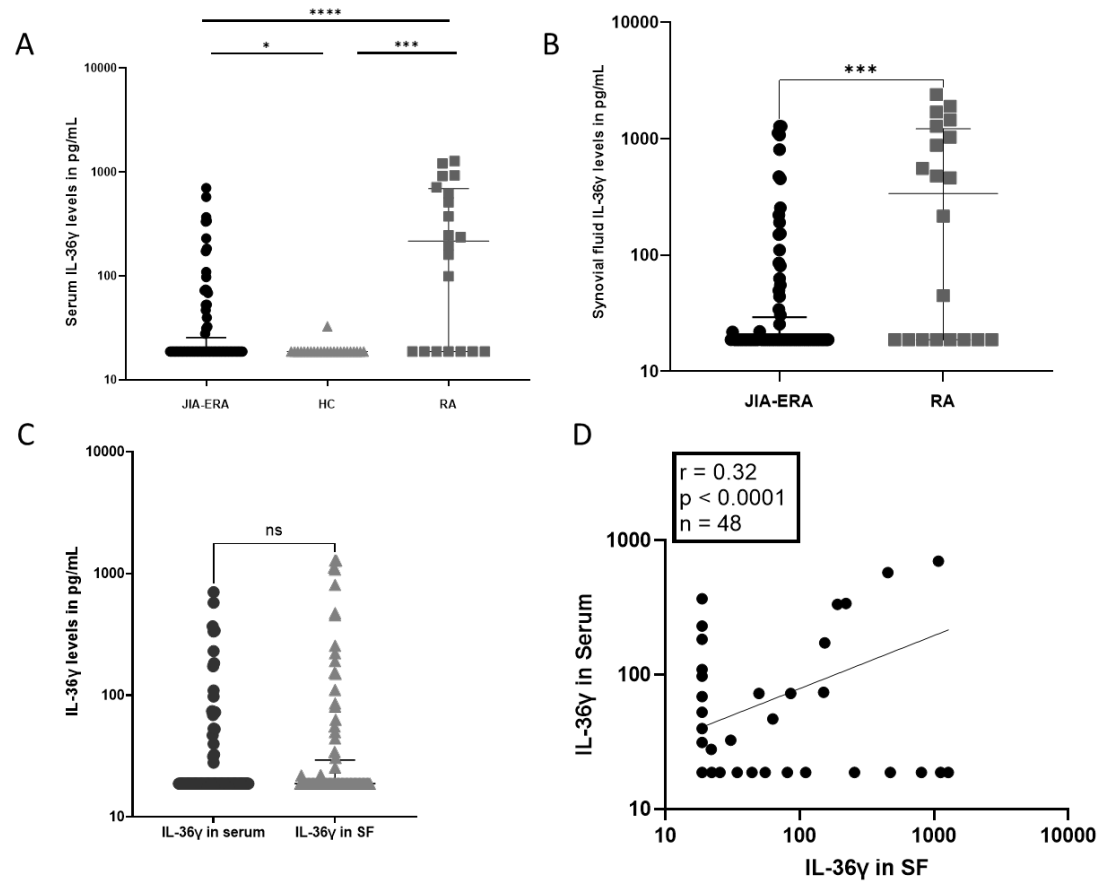

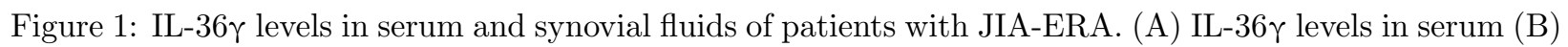
IL-36 $\gamma$ levels in synovial fluid (C) Correlation of IL-36r levels in serum and synovial fluid (D) Comparison of serum and synovial fluid levels of IL-36 $\gamma$. All data are represented as Median \pm IQR. Significance was determined by non-parametric Mann-Whitney U test $\left(*=\mathrm{p}[?] 0.05,{ }^{* * *}=\mathrm{p}[?] 0.001\right.$ and $\left.{ }^{* * * *}=\mathrm{p}[?] 0.0001\right)$.
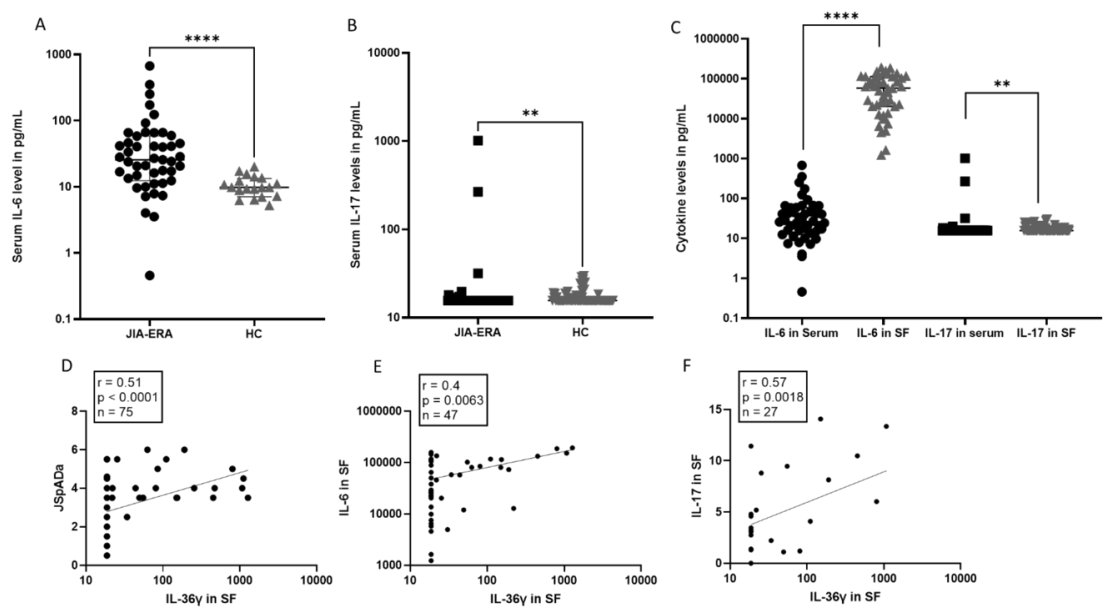

Figure 2: Correlation of IL-36r with disease activity and pro-inflammatory cytokine levels. (A) Serum IL-6 levels in patients with JIA-ERA compared to healthy control. (B) Serum IL-17 levels in patients with JIAERA compared to healthy control (C) Comparison of Serum and synovial fluid levels of IL-6 and IL-17 in patients with JIA-ERA (D) Correlation of synovial IL-36r levels with synovial IL-6 levels (E) Correlation of synovial IL-36 $\gamma$ levels with synovial IL-17 levels (F) Correlation of synovial IL-36 $\gamma$ levels with disease activity score JSpaDA. All data are represented as Median \pm IQR. Significance was determined by non-parametric Mann-Whitney $\mathrm{U}$ test $\left(*=\mathrm{p}[?] 0.05,{ }^{* * *}=\mathrm{p}[?] 0.001\right.$ and $\left.{ }^{* * * *}=\mathrm{p}[?] 0.0001\right)$. 

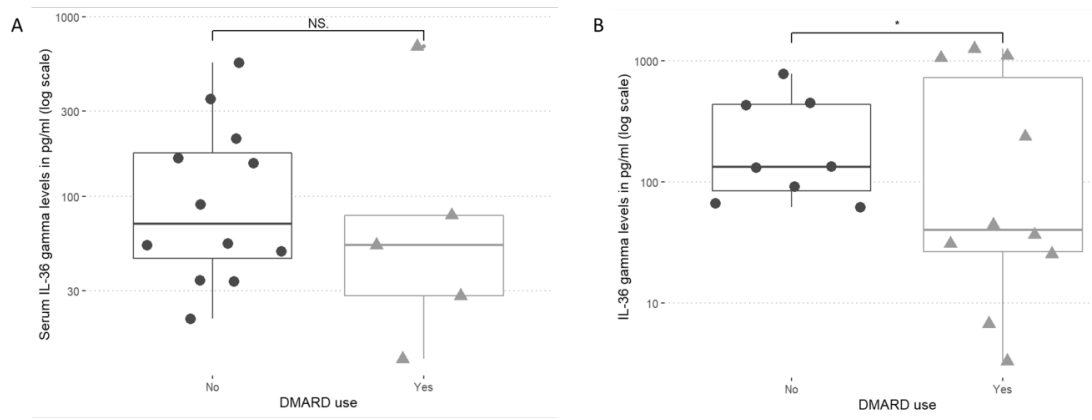

Figure 3: Serum and synovial IL-36 $\gamma$ levels in JIA-ERA patients undergoing treatment with DMARDs. (A) Comparison of synovial fluid IL-36r levels in JIA patients on DMARDs vs not on DMARDs (B) Comparison of serum IL-36r levels in JIA patients on DMARDs vs not on DMARDs. Significance was determined by non-parametric Wilcoxon signed-rank test $(*=\mathrm{p}[?] 0.05)$.
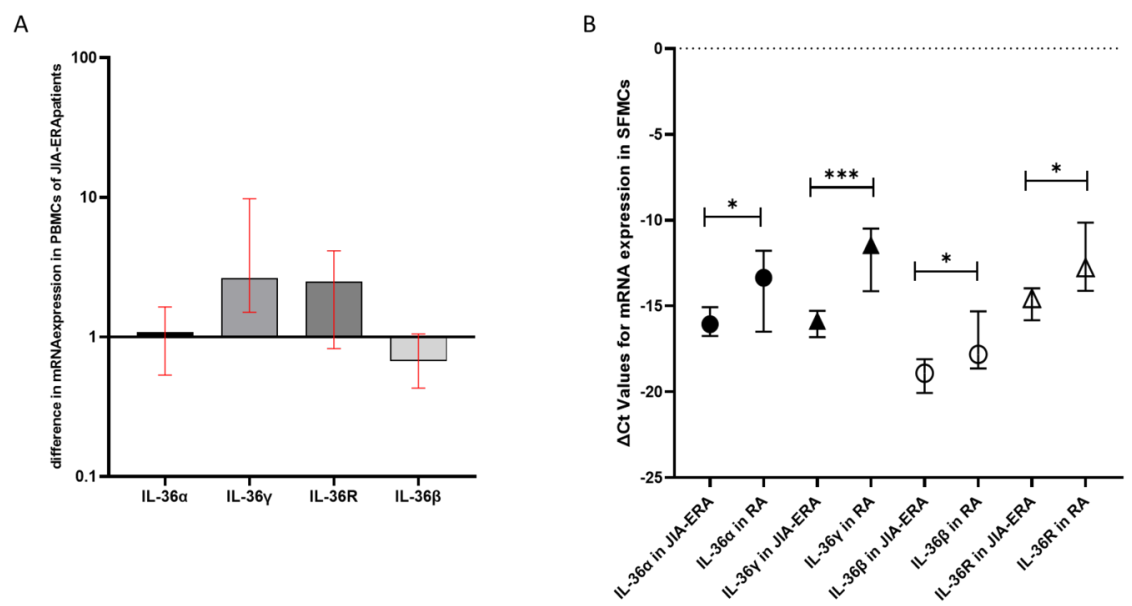

Figure 4: mRNA expression of IL-36 genes in PBMCs and SFMCs of patients with JIA-ERA. (A) fold difference in mRNA expression of IL-36 cytokine genes in PBMCs of JIA-ERA patients compared to healthy controls (B) $\Delta$ CT values depicting differences in mRNA expression of IL-36 genes in SFMCs of JIA-ERA patients compared to RA patients. All data are represented as Median \pm IQR. Significance was determined by non-parametric Mann-Whitney $\mathrm{U}$ test $\left(*=\mathrm{p}[?] 0.05,{ }^{* * *}=\mathrm{p}[?] 0.001\right)$. 

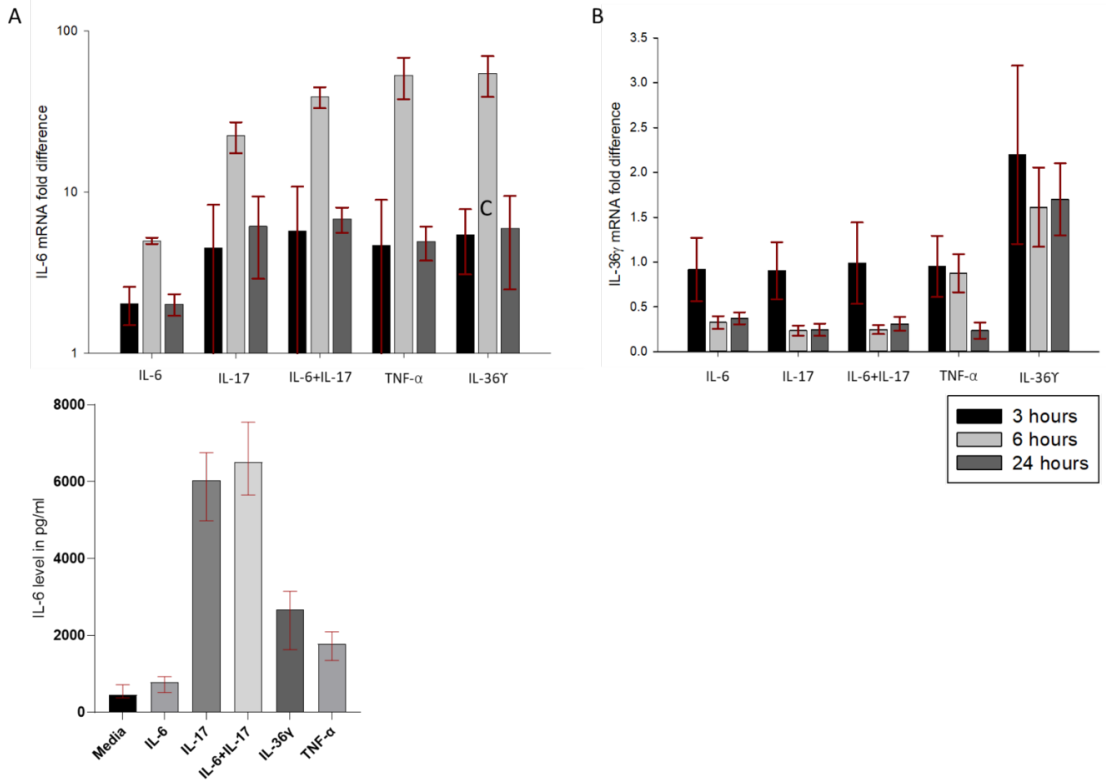

Figure 5: Expression of IL-36 $\gamma$ and IL-6 in FLS upon stimulation with pro-inflammatory cytokines (A) difference in mRNA expression of IL-6 in FLS upon stimulation with pro-inflammatory cytokines compared to unstimulated controls (B) difference in mRNA expression of IL-36 $\gamma$ in FLS upon stimulation with proinflammatory cytokines compared to unstimulated controls (C) IL-6 levels in FLS cell supernatant post stimulation with pro-inflammatory cytokines. All data are represented as Median \pm IQR.
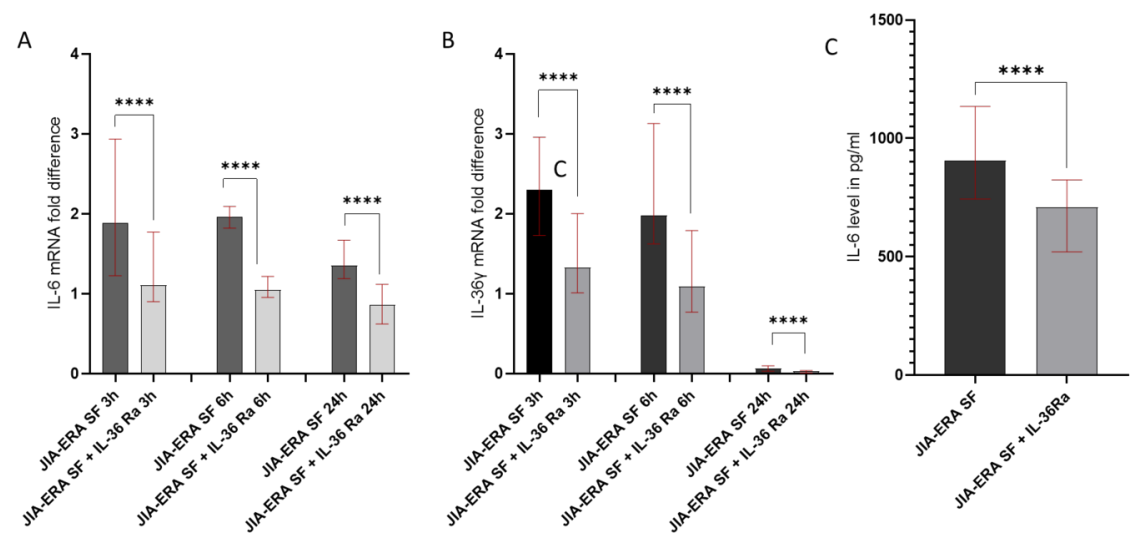

Figure 6: Expression of IL-36r and IL-6 in FLS upon stimulation with synovial fluids of patients with JIAERA in presence or absence of IL-36Ra (A) difference in mRNA expression of IL-6 in FLS upon stimulation with synovial fluids of patients with JIA-ERA in presence or absence of IL-36Ra (B) difference in mRNA expression of IL-36 $\gamma$ in FLS upon stimulation with synovial fluids of patients with JIA-ERA in presence or absence of IL-36Ra (C) IL-6 levels in FLS cell supernatant post stimulation with synovial fluids of patients with JIA-ERA in presence or absence of IL-36Ra. All data are represented as Median \pm IQR. Significance was determined by non-parametric Mann-Whitney U test $(* * * *=p[?] 0.0001)$. 